











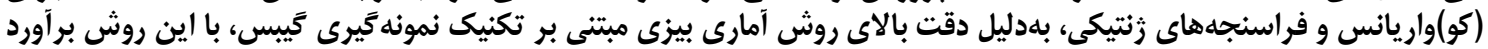

















وازهاى كليدى: روش بيزى، صفات رشد، فراسنجههاى زنتيكى، تَوسفند مغانى

بسيار مهم در توليد به حساب مى آيند و يكى از ابزارهاى مهارم




















روشهاى مونت كارلوى زنجيره ماركوف النيكرى (MCMC) است.









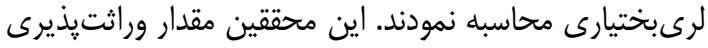

















مقدمه
















































محيطى را برآورد مى كند بود. تعيين عوامل محيطى مؤثر بر صفات رشد اين نزاد از ديخر اهداف اين بردوهش بردي بوداد



براى انجام اين بزوهش از اطلاعات شجرهاى و و ركوردهاى



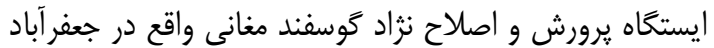

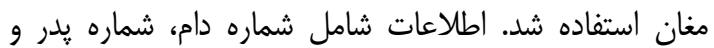

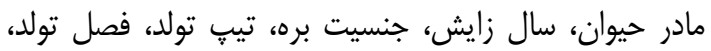

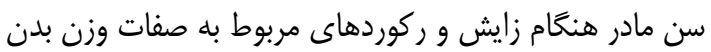

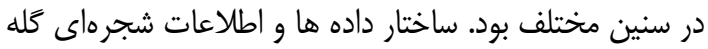
مورد مطالعه در جدول آورده شده است.
روش آمارى بيزى و تكنيك نمونهَيرى كيبس مقدار



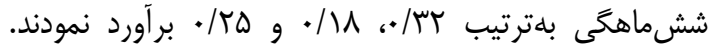
جاواسره' و همكاران (IV) در كَوسفندان آواسى، مقدار



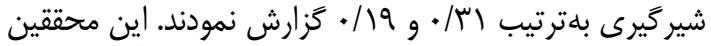



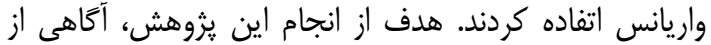

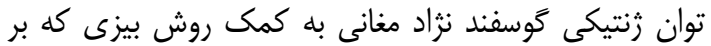

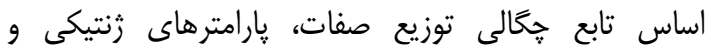

جدول ا - ساختار دادها و اطلاعات شجره اى گله گَوسفند مغانى مورد مطالعه Table 1. The data structure and pedigree information of the studied Moghani herd

\begin{tabular}{|c|c|c|c|}
\hline ت تعداد & اطلاعات & ت تعداد & اطلاعات \\
\hline $19 V$. & مادران غير ازنسل پايه & q)एव & كل حيوانات \\
\hline r & نتاج فقط داراى يدر & 1.49 & حيوانات هم خون \\
\hline$|M F|$ & نتاج فقط داراى مادر & ऍМq & كل يدرها \\
\hline 9191 & نتاج داراى پِدر و مادر & Ther & كل مادرها \\
\hline אזטו & كل اجداد & ᄉ19 & حيوانات موجود در فايل داده \\
\hline rar & يدر بزرگها & TAMT & حيوانات داراى نتاج \\
\hline$|T \Delta|$ & مادر بزركَها & $q^{\mu} \cdot V$ & حيوانات بدون نتاج \\
\hline Tr & يدربزركَهاى يدرى & qrT & حيوانات نسل پايه \\
\hline TVG & يدربزر گهاى مادرى & 1rq & يدران نسل يايه \\
\hline 1.14 & اجداد والدين & VAr & مادران نسل پايه \\
\hline rt. & اجداد يدرها & NTIV & حيوانات غير از نسل بايه \\
\hline v91 & اجداد مادرها &  & يدران غير ازنسل پايه \\
\hline
\end{tabular}

حافظه رايانه بستخى دارد. در برنامههاى MTGSAM براى






مى إتوانند استفاده شوند. نرمافزار

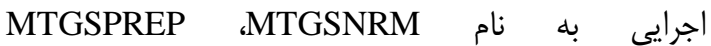
مى Mى باشد. براى شروع كار با نرمافزار ابتدا بايد

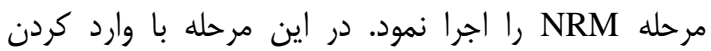

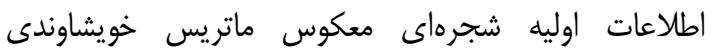
محاسبه مىشود تا از اين مقدار در مرحله بعد استفاده گردات مدرد.



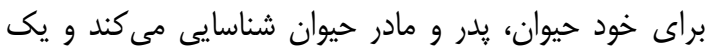

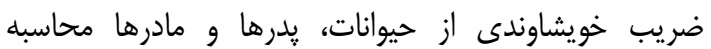
مى كند. در مرحله PREP اطلاعات اوليه مورد نظر از از جمله

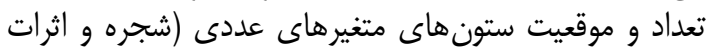



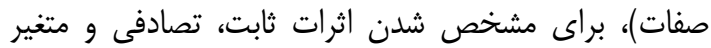

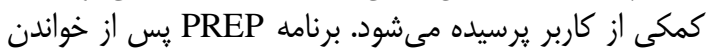

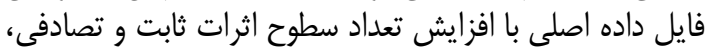



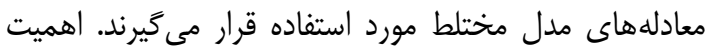


استفاده از رويه GLM نرمافزار آمارى









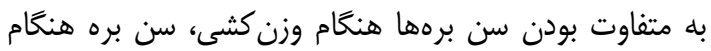

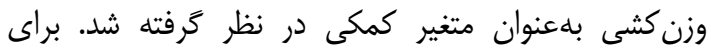

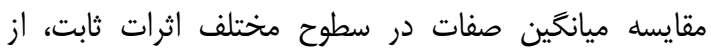

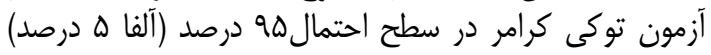

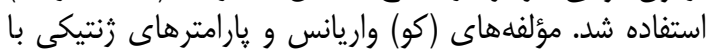
روش آمارى بيزى و نرمافزار

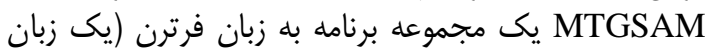

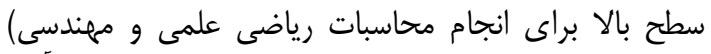

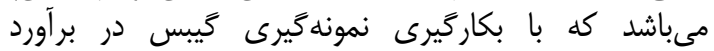

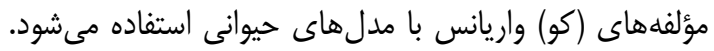

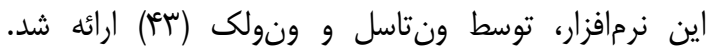



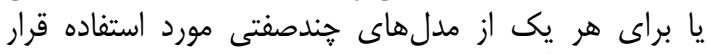

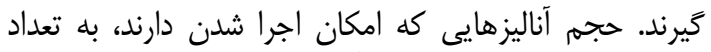

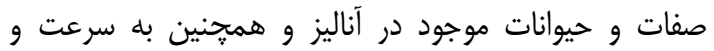




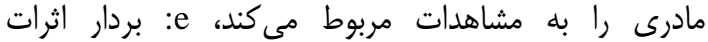

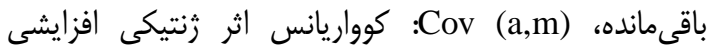

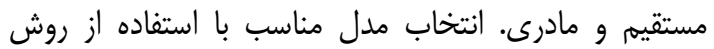

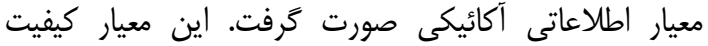

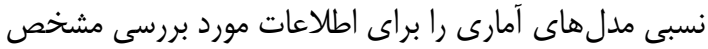

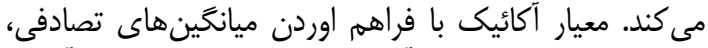

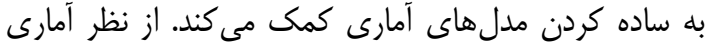

اين معيار صورت معادله زير محاسبه مى شیود (1).

$\mathrm{AIC}=\mathrm{n} \ln (\mathrm{RSS})+2 \mathrm{k}$

در اين رابطه RSS: مجموع مربعات باقيمانده، n: تعداد

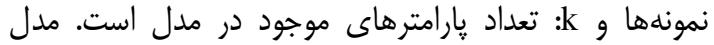

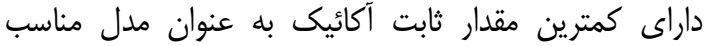

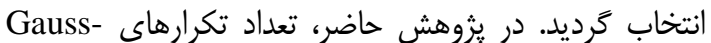
Seidel

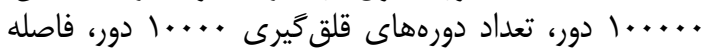

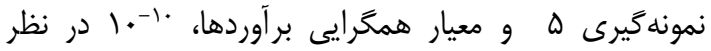
كرفته شدي وراثتيذيرى كل طبق فرمول زير محاسبه شد (هأ).

$$
h_{t}^{2}=\frac{\sigma_{a}^{2}+0.5 \sigma_{m}^{2}+1.5 \sigma_{a m}}{\sigma_{p}^{2}}
$$

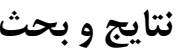

أمارههاى توصيفى صفات مورد بررسى در جدول r بن نشان

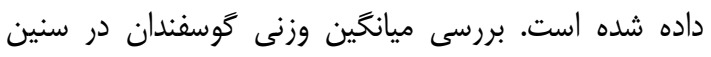

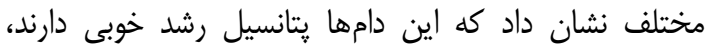

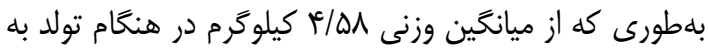

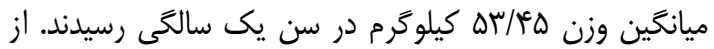

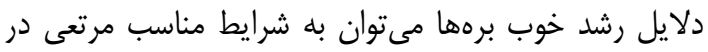

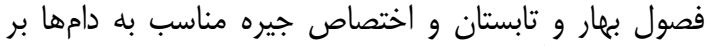
اساس شرايط آنها، اشاره كرد.

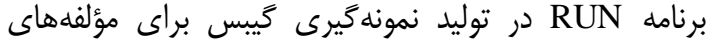

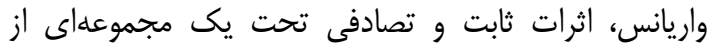

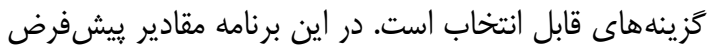

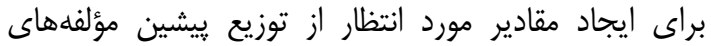

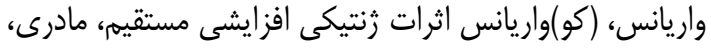

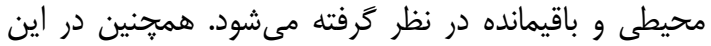

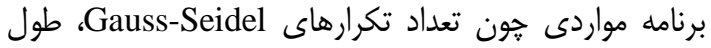

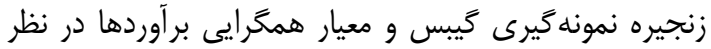

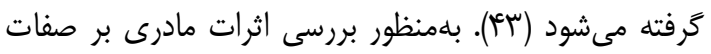

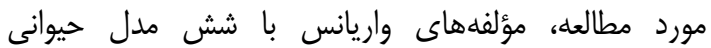
تكمتغيره مختلف برآورد شد (זَآ).

$\mathbf{y}=\mathbf{X b}+\mathbf{Z}_{\mathbf{1}} \mathbf{a}+\mathbf{e}$

$\mathbf{y}=\mathbf{X b}+\mathbf{Z}_{\mathbf{1}} \mathbf{a}+\mathbf{Z}_{\mathbf{2}} \mathbf{c}+\mathbf{e}$

$\mathbf{y}=\mathbf{X b}+\mathbf{Z}_{\mathbf{1}} \mathbf{a}+\mathbf{Z}_{\mathbf{3}} \mathbf{m}+\mathbf{e}$

$\operatorname{Cov}(\mathrm{a}, \mathrm{m})=0$

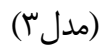

$\mathbf{y}=\mathbf{X b}+\mathbf{Z}_{\mathbf{1}} \mathbf{a}+\mathbf{Z}_{\mathbf{3}} \mathbf{m}+\mathbf{e}$

$\operatorname{Cov}(a, m)=A \sigma_{a m}$

$\mathbf{y}=\mathbf{X b}+\mathbf{Z}_{\mathbf{1}} \mathbf{a}+\mathbf{Z}_{\mathbf{2}} \mathbf{c}+\mathbf{Z}_{\mathbf{3}} \mathbf{m}+\mathbf{e}$

$\operatorname{Cov}(\mathrm{a}, \mathrm{m})=0$

$\mathbf{y}=\mathbf{X b}+\mathbf{Z}_{\mathbf{1}} \mathbf{a}+\mathbf{Z}_{\mathbf{2}} \mathbf{c}+\mathbf{Z}_{\mathbf{3}} \mathbf{m}+\mathbf{e}$

$\operatorname{Cov}(a, m)=A \sigma_{a m}$

y: بردار مشاهدات براى صفت مورد استفاده، b: بردات برار اثرات

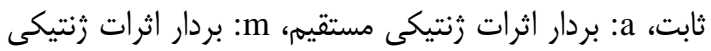

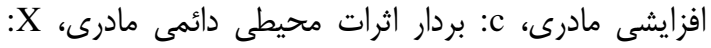

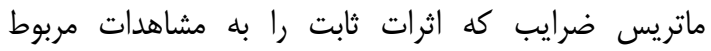
مى كند،

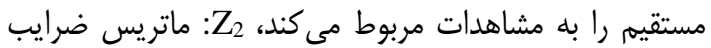

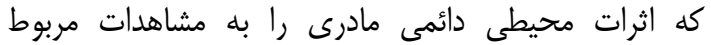
مى كند، Z3

جدول r- آمارههاى توصيفى صفات رشد (كيلوكَرم) در كوسفند مغانى Table 3. Descriptive statistics of growth traits (kg) in Moghani sheep

\begin{tabular}{|c|c|c|c|c|c|}
\hline وزن يكسالكى & وزن نdماهگى & وزن ششماهكى & وزن شيرگيرى & وزن تولد & اثر /صفت \\
\hline TMQT & rवार & D11. & Q१वY & 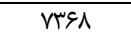 & تعداد كل \\
\hline$\Delta T / F \Delta \pm \cdot / T r$ & ऍ $/ \wedge ৭ \pm . /$. & $r \varphi / \Lambda \Delta \pm . / . q$ & $r \mu / q \mathrm{~V} \pm .1 .9$ & $\varphi / \Delta \wedge \pm \cdot / \cdot 1$ & ميانكَين كل \\
\hline $\mid 1 / \mu$ & $\Delta / V$. & g/rg & $\Delta / T r$ & ./VG & انحراف معيار \\
\hline$r / \Delta$ & r. & rו & 11 & $r / T$ & حداقل \\
\hline$V W / T$ & $\Delta \Delta / r$ & $\Delta N / I$ & $\varphi+/ \Delta$ & $V / 1$ & حداكثر \\
\hline WI. & س بא/.1 & $\mid f / M \Lambda$ & $10 / 1 T$ & $\mid r / T f^{c}$ & ضريب تغييرات \\
\hline
\end{tabular}

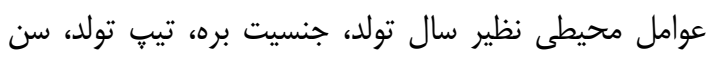
مادر هنكام زايش و كله قرار دار تولد

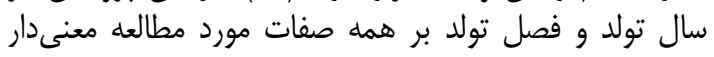

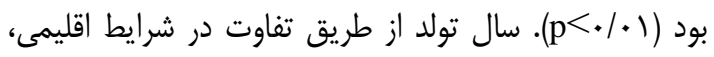

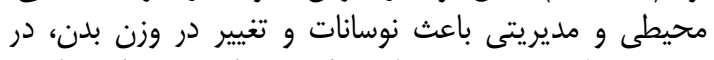

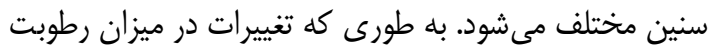

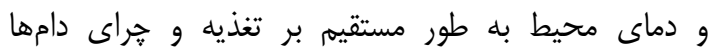

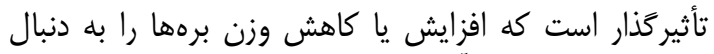

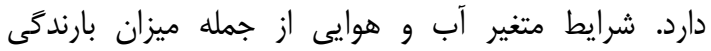

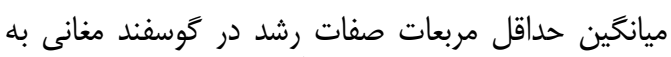

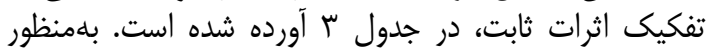

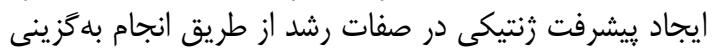

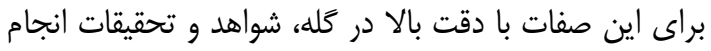

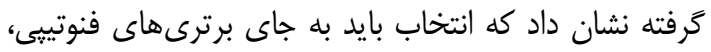

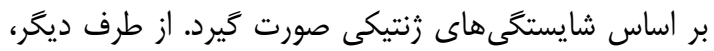

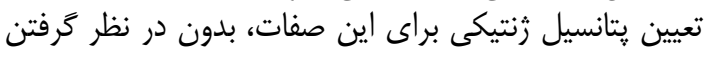

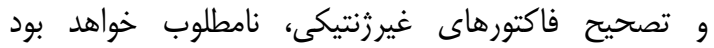

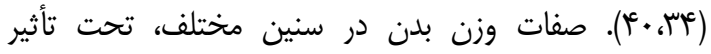


نتيحه تغذيه آنها محدودتر مىشود (أم). روبينسون (هَ)

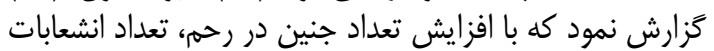

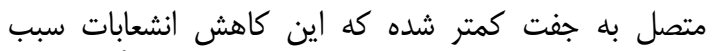

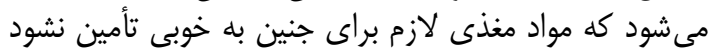

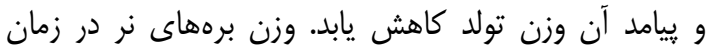

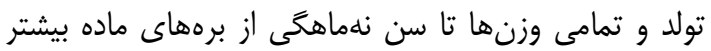

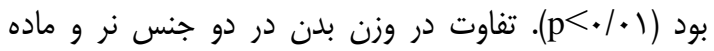

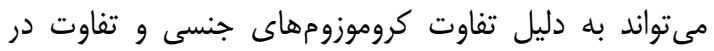

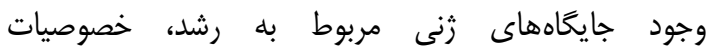

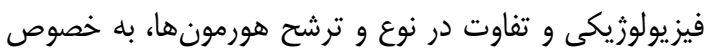

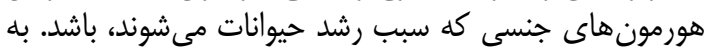

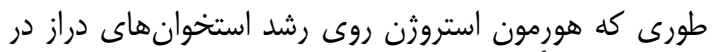

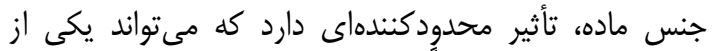

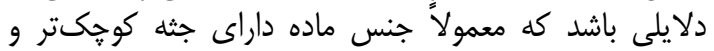

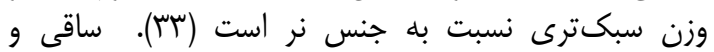

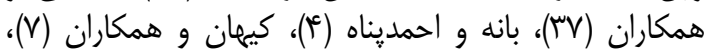

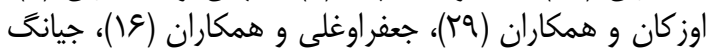

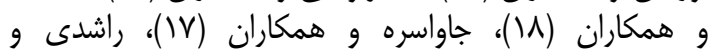

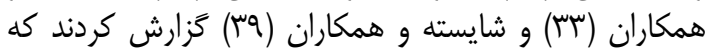

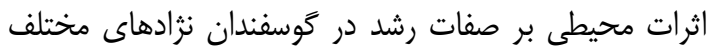

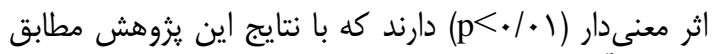

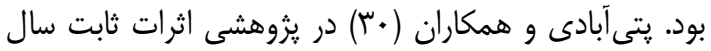

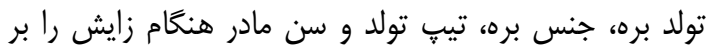

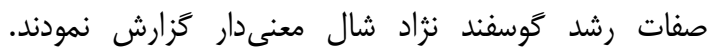

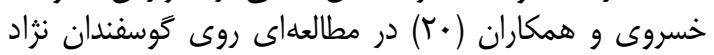

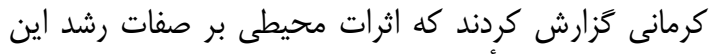

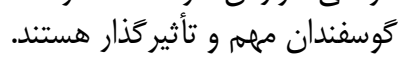

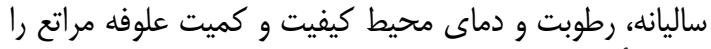

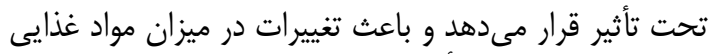

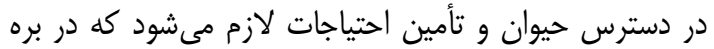

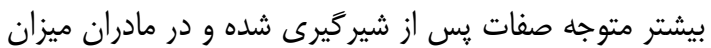

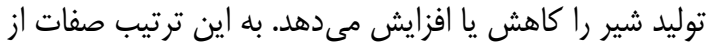

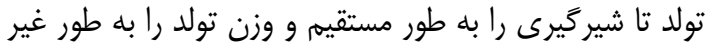

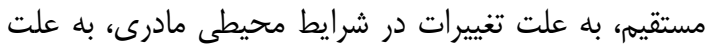

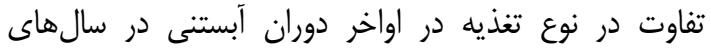

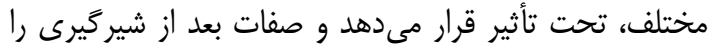

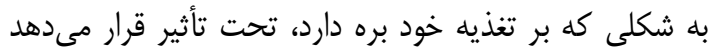

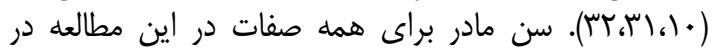

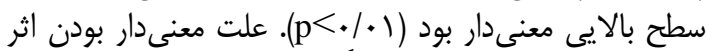

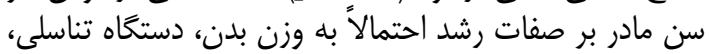

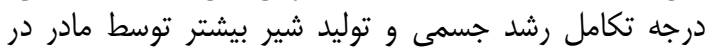

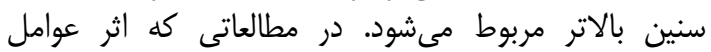

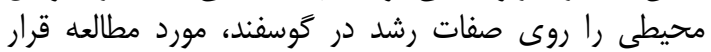

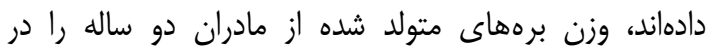

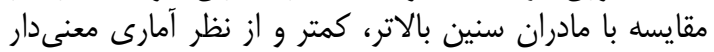

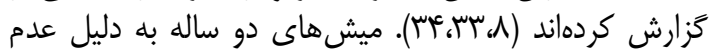

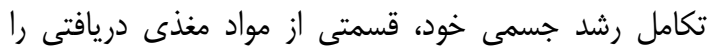

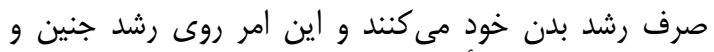

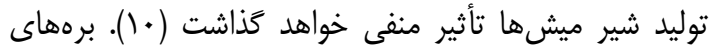

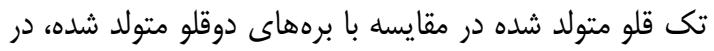

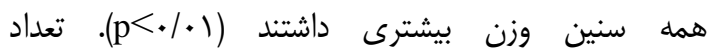

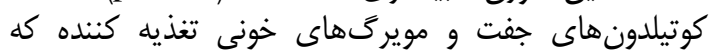

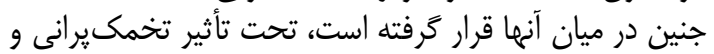

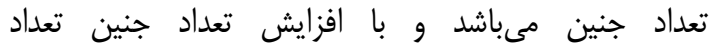

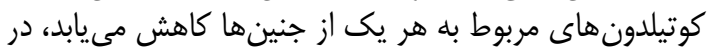

جدول ب- ميانگين حداقل مربعات صفات رشد (كيلوگرم) در گوسفند مغانى به تفكيك اثرات ثابت Table 3. Least squares Means of growth traits (kg) in Moghani sheep by constant fix effects

\begin{tabular}{|c|c|c|c|c|c|}
\hline وزن يكسالكى & وزن نهماهكى & وزن ششماهكى & وزن شيرگيرى & وزن تولد & اثر/صفت \\
\hline ** & $* *$ & ** & ** & $* *$ & سال تولد \\
\hline$* *$ & $* *$ & ** & $* *$ & $* *$ & سن مادر \\
\hline$\Delta r / T r \pm I / / r^{b}$ & $r q / \cdot r \pm \cdot / 8 q^{d}$ & $r q / q \vee \pm 1 / r \Lambda^{c}$ & $r \nu / \wedge \pm \cdot / l V^{b}$ & $r / \cdot V \pm \cdot / \cdot r d$ & r \\
\hline$\Delta r / / \Delta \pm 1 / 1 \cdot c$ & $r \Delta / \backslash \wedge \pm \cdot / 89^{d}$ & $r \cdot / ৭ q \pm 1 / r \Lambda^{c}$ & $T r / \& \notin \pm . / / V^{b}$ & $\left\ulcorner/ \leftarrow \wedge \pm . / . \tau^{b c}\right.$ & r \\
\hline$\Delta r / \Delta r \pm 1 / \cdot q^{a}$ & $r \Delta / V \Delta \pm \cdot / 89^{c}$ & $r / / ৭ q \pm 1 / \mu^{b}$ & $r r / Q \S \pm \cdot / 1 q^{a}$ & $\varphi / \Delta q \pm \cdot / \cdot r^{\mathrm{a}}$ & f \\
\hline$\Delta r / \cdot \wedge \pm 1 / / r^{a}$ & $r \Delta / \mathcal{N} \pm \pm \cdot / \varepsilon 9^{a}$ & $r \mid /{ }^{\prime} \wedge \pm 1 / q^{a}$ & $r r / \wedge \neg \pm \cdot / \wedge \Lambda^{\mathrm{a}}$ & $r / \Delta \Delta \pm \cdot / \cdot \mu c$ & $\Delta$ \\
\hline$\Delta F / T Y \pm 1 / / r^{\mathrm{ab}}$ & $r \Delta / r \checkmark \pm \cdot / V)^{b c}$ & $r / / q \vee \pm 1 / q^{a b}$ & $r r / q 9 \pm . / 19^{b}$ & $\varphi / \& \mu \pm . / . \mu a b$ & 9 \\
\hline$\Delta r /|q \pm| /\left.r\right|^{c}$ & $r e / v q \pm 1 / v^{a d}$ & $r \cdot / v a \pm 1 / r \cdot{ }^{a b}$ & C & $\leftarrow / \notin q \pm \cdot / \cdot r a$ & $v$ \\
\hline$* *$ & $* *$ & $* *$ & $* *$ & $* *$ & فصل تولد \\
\hline 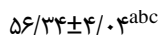 & $r v / \Lambda\rfloor \pm r / \lambda \omega^{b}$ & $r q / ৭ १ \pm \cdot / M^{c}$ & $r \cdot / V \subseteq \pm \cdot / r \Delta^{c}$ & $f / \mathcal{E} \cdot \pm \cdot / \cdot \Delta^{c}$ & 1 \\
\hline$\Delta N / Y \pm \pm / \cdot r^{\mathrm{a}}$ & -- & $r \varphi / \Lambda \& \pm \Psi / q \Lambda^{d}$ & $r \cdot / / V \pm \cdot / r Y^{d}$ & $\uparrow / \uparrow q \pm . / . q^{b c}$ & r \\
\hline$\Delta T / \cdot Y \pm \cdot / \Delta Y^{b}$ & 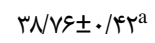 & $r g / Q \vee \pm . / r r^{a}$ & $r \Delta / f \mid \pm \cdot / \lambda \Lambda^{\mathrm{a}}$ & $\varphi / \Delta I \pm \cdot / \cdot r^{a}$ & r \\
\hline $\mid F / T Y \pm \cdot / r I^{c}$ & 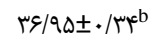 & $r F / ৭ \Lambda \pm . / .9^{b}$ & $r r / q \Lambda \pm . / . \vartheta^{b}$ & $\tau / \Delta \Delta \pm \cdot / \cdot 1^{\mathrm{ab}}$ & f \\
\hline$* *$ & $* *$ & $* *$ & $* *$ & $* *$ & تيب تولد \\
\hline$\Delta F / T V \pm 1 / \cdot \varphi^{\mathrm{a}}$ & $r q / ৭ q \pm \cdot / q \mathrm{~V}^{\mathrm{a}}$ & $r \Psi / \mathcal{} \Psi \pm 1 / \gamma^{\mathrm{a}}$ & $r \leftarrow / \wedge q \pm \cdot / / f^{c a}$ & $r / q \pm \pm \cdot / \cdot r^{\mathrm{a}}$ & تَك قلو \\
\hline$\Delta T / T^{\top} \pm 1 / \cdot \wedge^{\mathrm{b}}$ & $r \Psi / Q \Lambda \pm \cdot / 8 \Lambda^{\mathrm{a}}$ & $r \cdot / \Delta r \pm I / r \Lambda^{b}$ & $r / / r \Delta \pm \cdot / 1 \Delta^{b}$ & $r / \cdot q \pm \cdot / \cdot r^{b}$ & دوقلو \\
\hline ns & $* *$ & $* *$ & $* *$ & $* *$ & جنس بره \\
\hline$\Delta \% / \cdot \odot \pm 1 / \cdot \varphi^{\mathrm{a}}$ & $r V / \Lambda \varepsilon \pm \cdot / \varepsilon \Lambda^{\mathrm{a}}$ & $r \leftarrow / Q \Lambda \pm 1 / r \gamma^{a}$ & $r F / r \Lambda \pm \cdot / l)^{\mathrm{ca}}$ & $r / q \Lambda \pm \cdot / \cdot r^{\mathrm{a}}$ & نر \\
\hline$\Delta r /\left\{r \pm 1 / \cdot v^{a}\right.$ & $r \tau / Q \Lambda \pm \cdot / \varepsilon \Lambda^{\mathrm{b}}$ & $r \cdot / \uparrow 1 \pm 1 / r \gamma^{b}$ & $r / / \wedge V \pm \cdot / / f^{c b}$ & $r / r f \pm \cdot / \cdot r^{b}$ & ماده \\
\hline$-\cdot / \cdot \Delta \varphi^{* *}$ & . $/ \cdot \mu^{* * *}$ & $\cdot 11 \cdot 0^{* *}$ & $\cdot / l f f^{* *}$ & ----- & متغير كمكى سن بره به روز \\
\hline$\cdot / \Delta T$ &.$/ \Delta F$ &.$/ K^{2} T$ & $\cdot / \Delta \Delta$ & $. / 4 \mid$ & $\mathrm{R}^{2}$ \\
\hline
\end{tabular}


تحت تأثير عوامل زنتيكى متعددى قرار مى زيرند. در زمينه

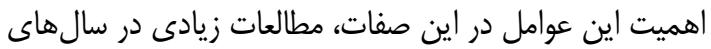

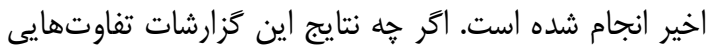

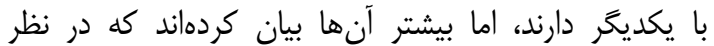

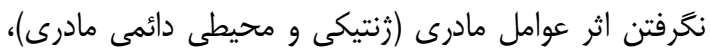

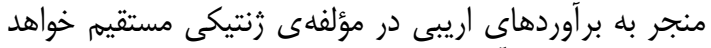

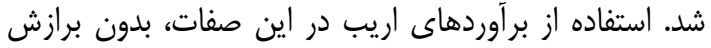

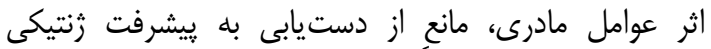

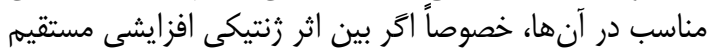

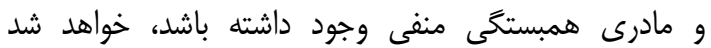

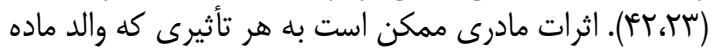

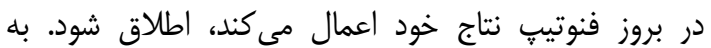
عنوان مثال مادر با توليد شير زياد، علاوه بر بر اينكا ائه اين استعداد

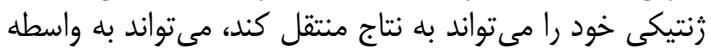

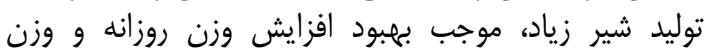

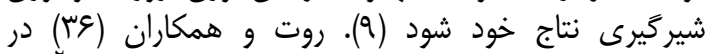

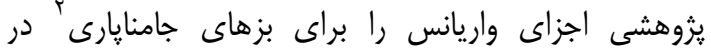

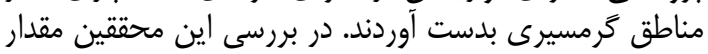

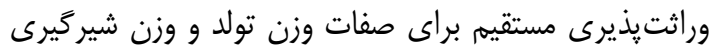

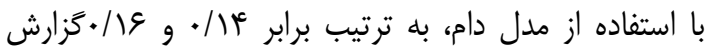

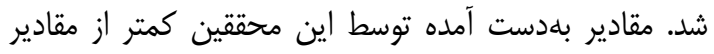

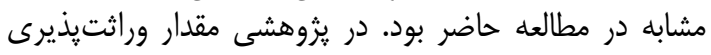

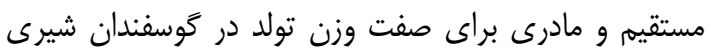

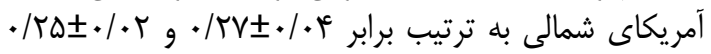

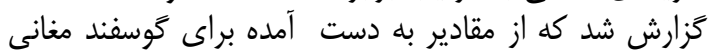

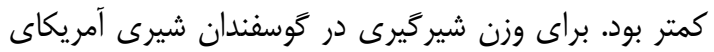

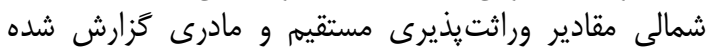

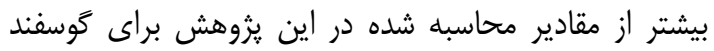

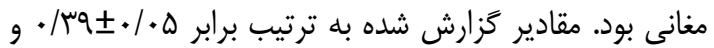

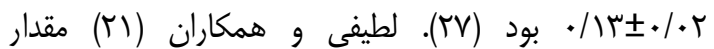

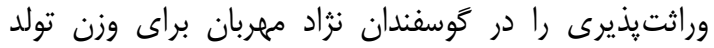

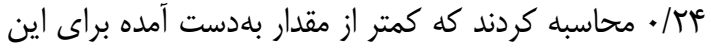

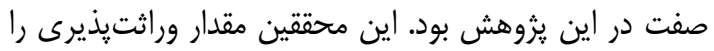

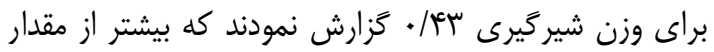

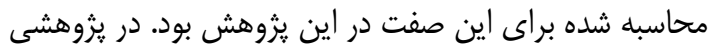

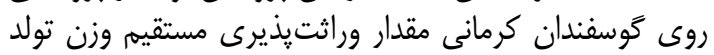

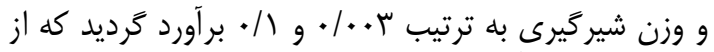

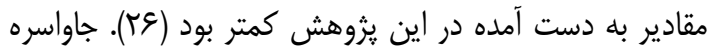

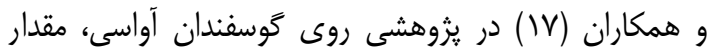

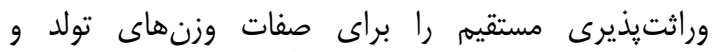

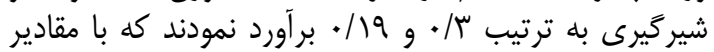
به دست آمده در اين بروهش مشابه نبود.

\section{برآورد اجزاى (كو)واريانس صفات رشد قبل از} جدول ع ب برآورد مدلهاى آمارى بر اساس معيار اطلاعات شير جيرى

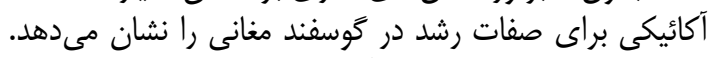

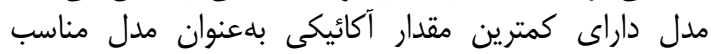

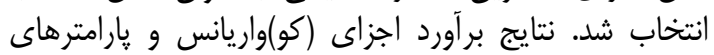

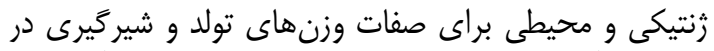

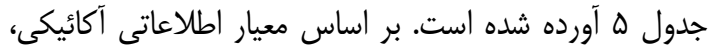

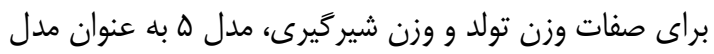

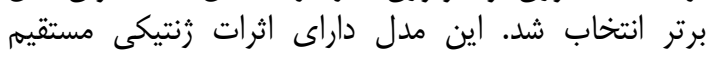

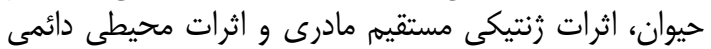

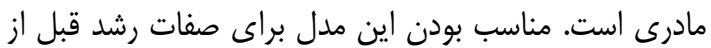

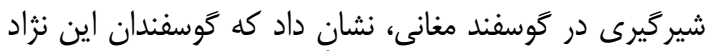

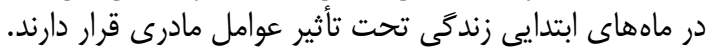

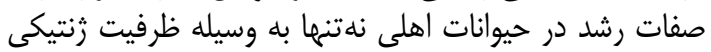

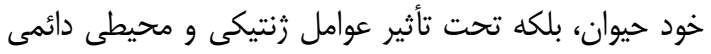

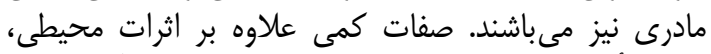

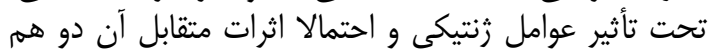

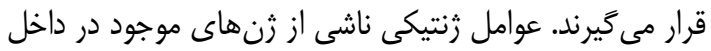

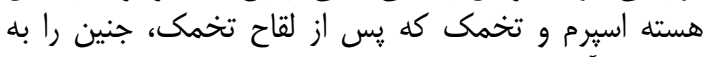

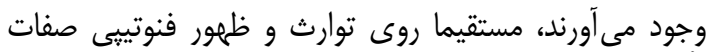

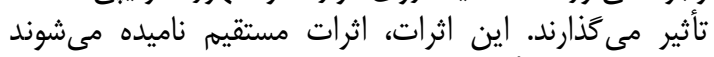

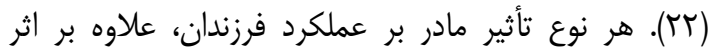

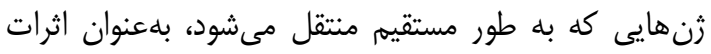

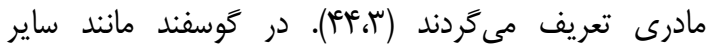

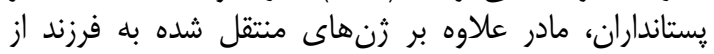

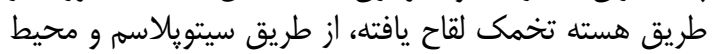

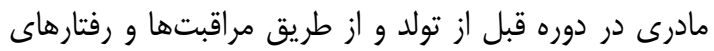

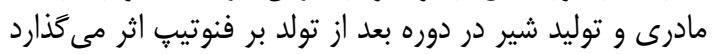

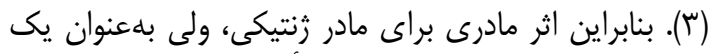

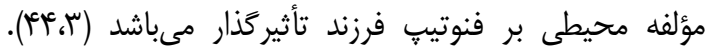

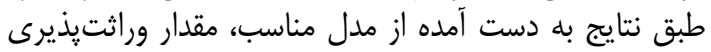

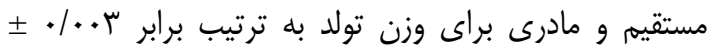

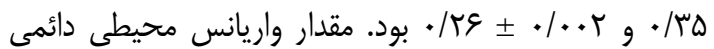

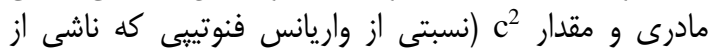

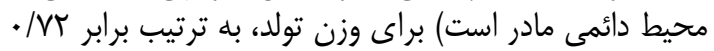

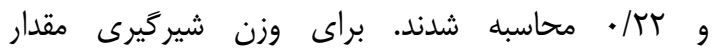

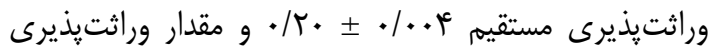

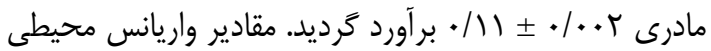

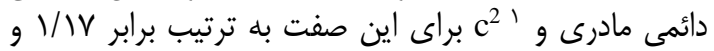

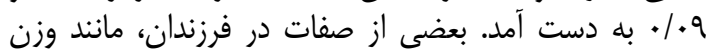

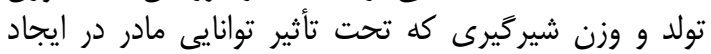

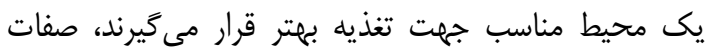
متأثر از مادر ناميده مىشوند. صفات توليدى در سنين مختلف، 
جدول أ - برآورد مدلهاى آمارى بر اساس معيار اطلاعات آكائيكى براى صفات رشد در گوسفند مغانى Table 4. The estimates of statistical models based on AIC for growth traits in Moghani sheep

\begin{tabular}{|c|c|c|c|c|c|c|}
\hline 9 & $\Delta$ & f & $r$ & $r$ & 1 & مدل \\
\hline$r$ & $r$ & $r$ & $r$ & $r$ & 1 & $\mathrm{k}$ \\
\hline -VNENF/KF & $-v 9111 / \Delta \wedge$ & -VAYTYA/IS & -VATYYA/IS & $-V \Lambda S \Lambda S / Y F$ & -VNSMNTF & وزن تولد \\
\hline -rVQYN/FA & $-r V q Q V / q F$ & $-T V V q T / V T$ & $-r V V G M / V T$ & $-r V \Delta \wedge / / Q \varepsilon$ & -rVQqTT/FA & وزن شيرگيرى \\
\hline - TMMT/Vq & $-r M \subseteq \Phi / \Delta$. & - TAVAF/NE & 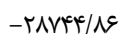 & $-r q .10 / 9 q$ & $-r \wedge q \Delta \backslash / r q$ & وزن عماهكى \\
\hline-1 Isqv/NT & $-1990 \cdot / 9)^{\circ}$ & $-\mid \xi \Delta \& D / T \Lambda$ & $-1 \xi \Delta ৭ \uparrow / \digamma$. & $-19 V+.1 .$. & $-199 \Delta f / 95$ & وزن أماهكَى \\
\hline 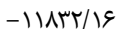 & -IIATE/rV & $-11 \Lambda T \omega / \uparrow \Lambda$ & $-11 \wedge \mu y / 19$ & - IINFNGT & $-11 \wedge r \cdot / r V$ & وزن يكسالكى \\
\hline
\end{tabular}

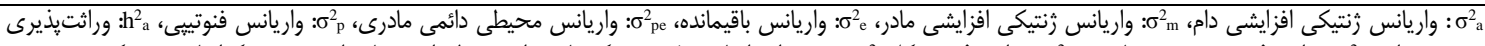

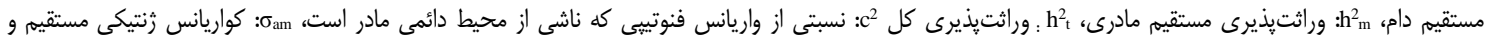

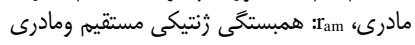

جدول ه- بر آورد اجزاى (كو)واريانس و يارامترهاى زنتيكى صفات رشد قبل از شير گيرى در گَوسند مغانى Table 5. Estimation of Components (Co) of Variance and Genetic Parameters of Growth Traits at pret-weaning in

\begin{tabular}{|c|c|c|c|c|c|c|c|}
\hline .119 & .119 & •/Tr & • & --- & --- & $\sigma_{\mathrm{m}}^{2}$ & \\
\hline$\cdot / \mathrm{V}$ &.$/ 19$ & $\cdot / \Lambda \Lambda$ & $\cdot / \Lambda \Lambda$ & $\cdot / \mathrm{V}$ &.$/ 1 V$ & $\sigma_{\mathrm{e}}^{2}$ & \\
\hline$\cdot N T$ & $\cdot N r$ & $\cdot N r$ & $\cdot N$ & $\cdot M r$ & $\cdot / N$ & $\sigma_{p}^{2}$ & وزن تولد \\
\hline$\cdot / \mu \pm \pm \cdot \cdot \cdot r$ & $\cdot / \Gamma \Delta \pm+* \cdot \cdot r$ & . & $. / \mu e_{ \pm} \cdot / \cdot r$ & $\cdot / r q_{ \pm} \cdot / \cdot \cdot r$ & 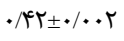 & $\mathrm{h}^{2} \mathrm{a}$ & \\
\hline$\cdot / \pi V$ &.$/ 4 \lambda$ & - &.$/ 19$ & • & . Mt & $h^{2}$ & \\
\hline.$- / \cdot 0$ & --- & $-\cdot 1 \cdot \Delta$ & --- & --- & -- & $\sigma_{\mathrm{am}}$ & \\
\hline س & --- & -- & --- & --- & -- & $\mathrm{r}_{\mathrm{am}}$ & \\
\hline$\cdot / T T \pm+1++1$ & $\cdot / T_{ \pm}+/ \cdot+1$ & --- & --- & r.•/.* & --- & $c^{2}$ & \\
\hline$r / 9 q$ & $r / \& \Lambda$ & $r / g F$ & $r / g F$ & $r / \Delta F$ & سא/א & $\sigma_{a}^{2}$ & \\
\hline Ir/K & Ir/TV & Ir/r & $1 \mathrm{I} / \mathrm{r}$ & IT/Kr & $1 \pi / 9 r$ & $\sigma_{\mathrm{p}}^{2}$ & شيرگيرى وزن \\
\hline$\cdot / 19 \pm * / * \varphi^{4}$ & $\cdot / r \cdot \pm * / \cdot \cdot f$ & $\cdot / 19 \pm * / * r$ & $\cdot / 19 \pm * / \cdot r$ & $\cdot / T V \pm \cdot / \cdot \cdot r$ & $\cdot / \uparrow \varepsilon_{ \pm \cdot * / \cdots \Delta}$ & $\mathrm{h}^{2} \mathrm{a}$ & \\
\hline 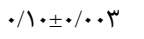 & $. / N \pm * . \cdot+r$ & $. / 19 \pm * / * r$ & $. / 19 \pm * / \cdot r$ & --- & --- & $\mathrm{h}^{2}{ }_{\mathrm{m}}$ & \\
\hline$\cdot / r$ & $\cdot / T \Delta$ & $\cdot / T \Delta$ &.$/ 49$ & $\cdot / T V$ & • & $\mathrm{h}^{2} \mathrm{t}$ & \\
\hline שוא/•- & --- & ( & --- & --- & --- & $\sigma_{\mathrm{am}}$ & \\
\hline.$- / 18$ & --- & -- & --- & --- & --- & $\mathrm{r}_{\mathrm{am}}$ & \\
\hline$* / \cdot \Lambda \pm * / *+1$ & $. / \cdot 9 \pm * 1+.1$ & --- & --- & •/IVE*/..r & --- & $c^{2}$ & \\
\hline
\end{tabular}

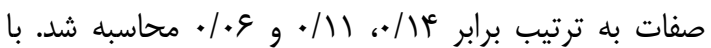
افزايش سن بهعلت كاهش وابستخى بره بله مادر انتظار مىرود

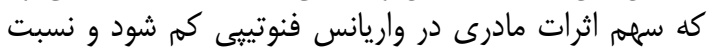

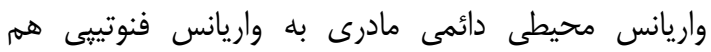

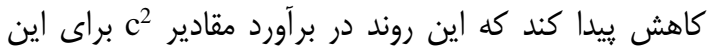

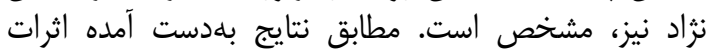

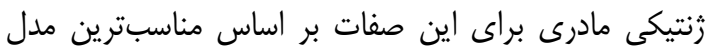

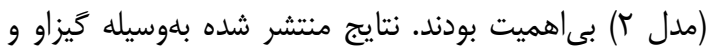

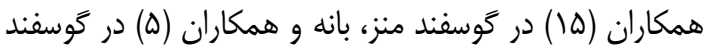

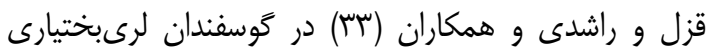

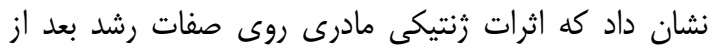

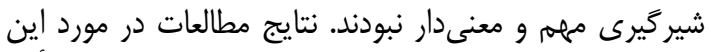

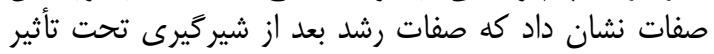

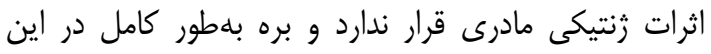

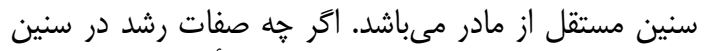

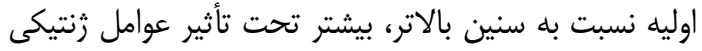

برآورد اجزاى (كو)واريانس صفات رشد بعد از شير تيرى

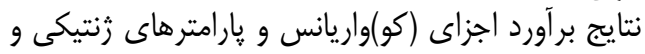

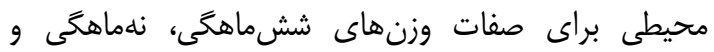

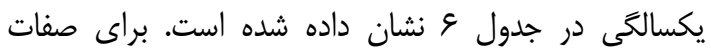

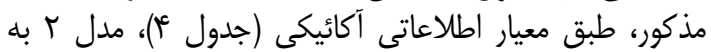

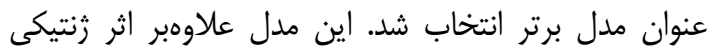

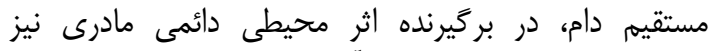

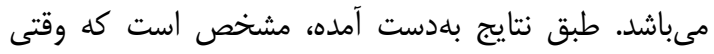

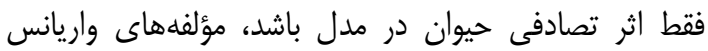

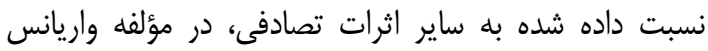

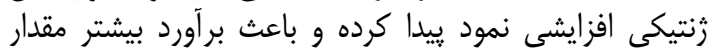

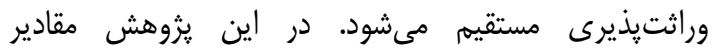

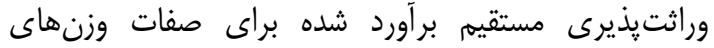

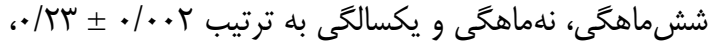

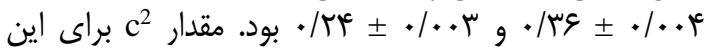




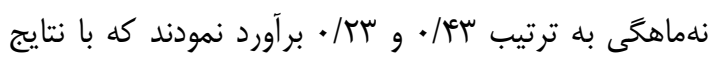

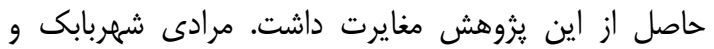

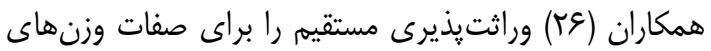

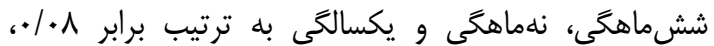

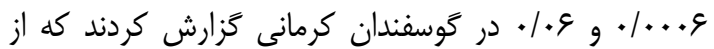

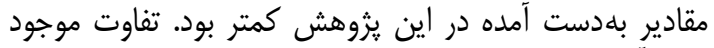

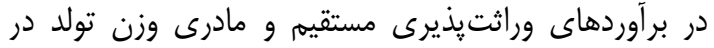

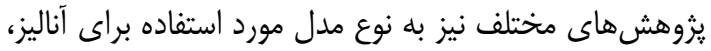

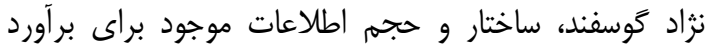

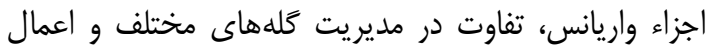

برنامههاى اصلاح نزادى متفاوت بستخى دارد (I) (I).

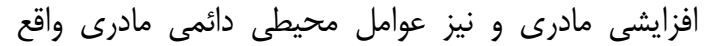

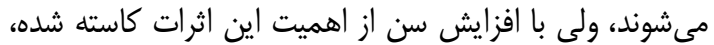

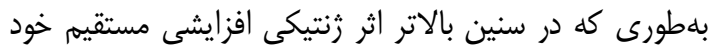

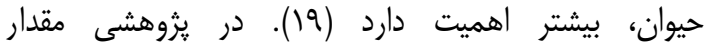

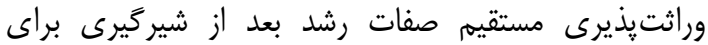

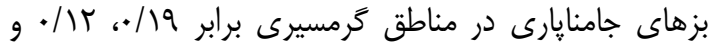

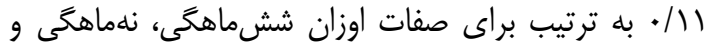

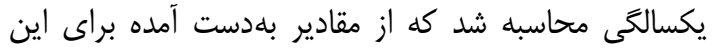

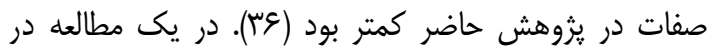

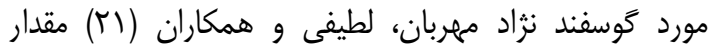

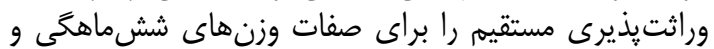

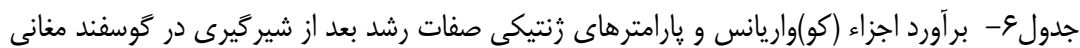
Table 6. Estimation of Components (Co) of Variance and Genetic Parameters of Growth Traits at post-weaning in

\begin{tabular}{|c|c|c|c|c|c|c|c|}
\hline مدل 9 & مدل ه مد & مدل F & مدل r & مدل r & مدل 1 & اجزاء/مدل & صفت \\
\hline$F / \Delta T$ & $F / V$ & $F / \Delta \Delta$ & $r / q V$ & $9 / \cdot 1$ & $N / \cdot r^{f}$ & $\sigma_{a}^{2}$ & \\
\hline r/\%q & T/KT & $T / M^{c}$ & T/Vq & -.- & --- & $\sigma_{\mathrm{m}}^{2}$ & \\
\hline אוא & $r / \Lambda \Delta$ & --- & --- & $r / 91$ & --- & $\sigma_{\mathrm{pe}}^{2}$ & \\
\hline $1 N \cdot 9$ & $\mathrm{~W} \cdot \mathrm{r}$ & $W / F^{2} V$ & INAT & $\mid V / \& q$ & $\mid V / 9 \Lambda$ & $\sigma_{e}^{2}$ & \\
\hline T & TI/DT & $r q / g V$ & $r g / 8 \Lambda$ & Tr/ & 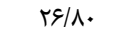 & $\sigma_{p}^{2}$ & وزن و ماهخى \\
\hline$\cdot / \mathrm{V} \pm * / \cdot \cdot 1$ & $\cdot / \Lambda \Lambda \pm * / *)$ & $\cdot / \mathrm{V} \pm * / \cdot \cdot r$ & $\cdot / \Lambda \pm \cdot / \cdot \cdot \mid$ & $\cdot / r \pm \pm \cdot / \cdot r$ & $\bullet / T_{ \pm}+/ \cdot \Delta$ & $\mathrm{h}^{2} \mathrm{a}$ & \\
\hline$\cdot / \cdot 9 \pm * / \cdot \cdot r$ & $\bullet \cdot q_{ \pm} \cdot / \cdot \bullet$ & $\cdot / 1 \cdot \pm * / \cdot * r$ & $\cdot / \cdot \pm \cdot / \cdot r$ & --- & --- & $\mathrm{h}_{\mathrm{m}}^{2}$ & \\
\hline.$/ 11$ & trt &.$/ \mathrm{V}$ & זr/. & r & $\cdot \pi$ & $\mathrm{h}_{\mathrm{t}}^{2}$ & \\
\hline.$- / q \mu$ & --- &.$- / 9 \pi$ & --- & --- & --- & $\sigma_{\mathrm{am}}$ & \\
\hline.$- / I V$ & --- & -- & -- & --- & --- & $\mathrm{r}_{\mathrm{am}}$ & \\
\hline$\cdot / \cdot \Lambda \pm \cdot / \cdot r$ & $\cdot / 1 \pm \cdot / \cdot r$ & -- & --- & $\cdot / f_{ \pm} \cdot / \cdot \cdot r$ & -- & $c^{2}$ & \\
\hline$F / \Delta \Delta$ & $F / \Delta \Delta$ & $F / 4$ & $F / \Delta$. & $\Delta / \Delta V$ & $s / r q$ & $\sigma_{a}^{2}$ & \\
\hline $4 / 11$ & $r / l f$ & $T / T)$ & $T / T F$ & --- & --- & $\sigma_{\mathrm{m}}^{2}$ & \\
\hline $1 / V^{e}$ & $1 / V \Delta$ & -- & -- & $r / \cdot 1$ & -- & $\sigma_{\mathrm{pe}}^{2}$ & \\
\hline$q / \Delta \rho$ & $q / \Delta T$ & $q / v \wedge$ & $q / V^{c}$ & $q / \Gamma \Delta$ & $q / \Delta \Delta$ & $\sigma_{e}^{2}$ & \\
\hline IV/VQ & IV/VQ & IV/DT & $I V / \Delta T$ & $1 V / r q$ & $I V / \& V$ & $\sigma_{p}^{2}$ & وزن 9 ماهكى \\
\hline$. / \Gamma \varphi_{ \pm} \cdot / \cdot r$ & $\cdot / r \varepsilon_{ \pm} \cdot / \cdot \cdot r$ & $\cdot / r \Delta \pm \cdot / \cdot r r$ & $\cdot / r \varphi_{ \pm} \cdot / \cdot \cdot F$ & $\cdot / T_{ \pm} \cdot / \cdot \cdot r$ & $\cdot / q_{ \pm} / \cdot \cdot r$ & $\mathrm{~h}^{2} \mathrm{a}$ & \\
\hline$. / 1 \tau \pm \cdot / \cdot \cdot 1$ & $. / r \pm * / \cdot r$ & $. / r \pm \cdot / \cdot .1$ & $\cdot / / \mu_{ \pm} \cdot / \cdot r r$ & ..- & -.- & $\mathrm{h}^{2} \mathrm{~m}$ & \\
\hline$\cdot / \Gamma$. & TH & . & Tr/ & Tr/. & צמאן & $\mathrm{h}_{\mathrm{t}}^{2}$ & \\
\hline$-\cdot|r|$ & -- &.$- / 19$ & --- & --- & --- & $\sigma_{\mathrm{am}}$ & \\
\hline.$- / 8$ & -.- & -- & --- & -.- & -.- & $\mathrm{r}_{\mathrm{am}}$ & \\
\hline$\cdot / \cdot \pm \cdot / \cdot \cdot r$ & $\cdot / \cdot \pm \cdot / \cdot \cdot r$ & -- & -- & $\cdot / 1 \pm \cdot / \cdot \bullet r$ & --- & $c^{2}$ & \\
\hline $10 / 11$ & ID/Tr & $10 / 1$ & $1 \omega / T$ & $18 / 91$ & $18 / 90$ & $\sigma_{a}^{2}$ & \\
\hline s/V & $8 / V 9$ & S/M & $8 / 9$. & --- & -.- & $\sigma_{\mathrm{m}}^{2}$ & \\
\hline$r / q \mu$ & $r / q 4$ & -.- & --. & ए/Q८ & --. & $\sigma_{\mathrm{pe}}^{2}$ & \\
\hline$F N / T V$ & FNMT & $\lceil N / \uparrow \Lambda$ & $F N / F F$ & FN/ & $\uparrow N / \mu$ & $\sigma_{e}^{2}$ & \\
\hline $9 q / 14$ & $99 / 14$ & $99 / \mathrm{v}$ & $s q / v \wedge$ & $99 / \mathrm{V}$ & $99 / V$ & $\sigma_{\mathrm{p}}^{2}$ & وزن يكسالكى \\
\hline$\cdot / T_{ \pm}+\|^{\prime} \cdot T^{r}$ & $\cdot / T_{ \pm} \cdot / \cdot \cdot r$ & ש.*/RTE & $\cdot / T T \pm \cdot / \cdot r^{\varepsilon}$ & $\cdot / r t \pm \cdot / \cdot r$ & $\cdot / r \pm \pm \cdot / \cdot r$ & $\mathrm{~h}^{2}{ }_{\mathrm{a}}$ & \\
\hline$\cdot 11 \cdot \pm \cdot 1 \cdot \cdot 9$ & $\cdot / 1 \cdot \pm \cdot 1 \cdot+\Delta$ & $\cdot / 1 \cdot \pm \cdot / \cdot+r$ & $\cdot / 1 \cdot \pm \cdot / \cdot+r$ & --- & --- & $\mathrm{h}_{\mathrm{m}}^{2}$ & \\
\hline$\cdot / \mathrm{VV}$ & $\cdot / T V$ & $\cdot / \mathrm{IV}$ & $\cdot / T V$ & $\cdot / r^{r}$ & $\cdot / \pi r$ & $\mathrm{~h}^{2} \mathrm{t}$ & \\
\hline$-r / \mu r$ & -- & $-F / \mu$ & --- & --- & --- & $\sigma_{\mathrm{am}}$ & \\
\hline$-\cdot / 4 \mid$ & --- & -- & --- & --- & --- & $\mathrm{ram}_{\mathrm{am}}$ & \\
\hline $.1 .9 \pm .1 \cdot .+$ & $.1 \cdot \varphi_{ \pm} \cdot / \cdot \cdot r$ & --- & --- & $.1 \cdot 9 \pm . / \cdot r$ & -- & $c^{2}$ & \\
\hline
\end{tabular}

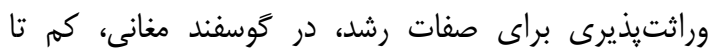

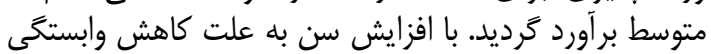

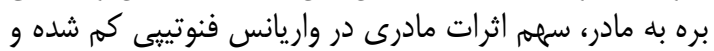

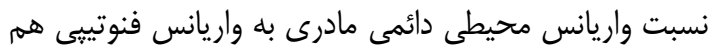

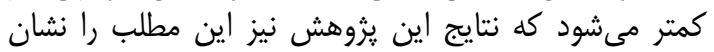

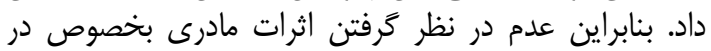

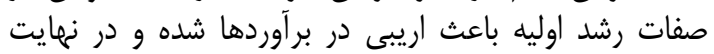
ياسخ به انتخاب را تحت تأثير قرار خواهد دادي
نتايج اين تحقيق نشان داد كه صفات رشد در كوسفند

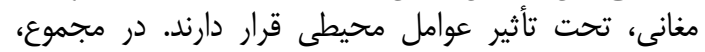

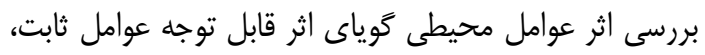

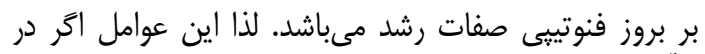

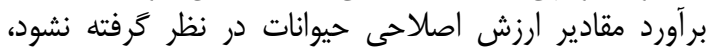

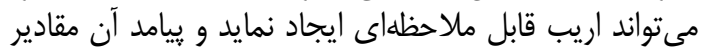

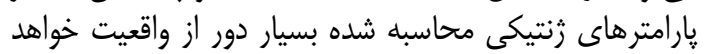
بود. بر اساس نتايج بهدست آمده در اين يُزوهش، مقدار 
1. Akaike, H. 1983. Information measures and model selection. Proceedings of the $44^{\text {th }}$ session of the internationalstatistical institute, 1: 277-291.

2. Azizi, P., M. Aali, M. MoradiShahrbabak, H. MoradiShahrbabak and H. Moghbeli. 2014. Estimation of genetic parameters and genetic trends for pre-weaning traits in Zel sheep. Journal of Livestock research, 4: 71-80 (In Persian).

3. Bahrini- Behzadi, M.R., F. Eftekhari Shahroudi and D. Van Volek. 2005. Maternal effect traits on heritability estimation and determination of environmental factors affecting on early growth traits in Kerman sheep. Journal of Agricultural Science and Natural Resources, 9: 195-2-2 (In Persian).

4. Baneh, H. and J. Ahmadpanah. 2018. Genetic evaluation of body weight traits in Iranian Native Ghezel sheep. Journal of Genetic, 50(1): 275-284.

5. Baneh, H., S.H. Hafezian., A. Rashidi and M. Gholizadeh. 2010. Estimation of genetic parameters of body weight traits in Ghezel sheep. Asian-Aust. Journal of Animal Science. 23: 149-153.

6. Baneh, H., E. Hashemi, M. Abbasi, F. Ghaforicasbi and B. Soleimani.2011. The study of genetic andphenotypic parameters for some of growth traitsinGhezel sheep. Congress fourth animal science. TehranUniversity, 3384-3388 (In Persian).

7. Ceyhan, A., T. Sezenler and I. Erdogan. 2009. The estimation of variance components for prolificacy and growth traits of Sak1z sheep. Livestock Science, 122: 68-72.

8. Dixit, S.P., J.S. Dhillon and G. Singh. 2001. Genetic and non-genetic parameters for growth traits of Bharat Merino lambs. Small Ruminant Research, 42: 101-104.

9. Dugoma, G., S.J. Schoeman., S.W.P. Cloete and G.F. Jordaan. 2002. Genetic parameter estimate of early growth traits in the Tygerhoek Merino flock. S. African Journal of Animal Science, 32(2): 6675.

10. Eftekhari Shahroudi, F., M.R. Bahrini, D. Ven Doulk and M. Danesh Mesgaran. 2002. The factor affecting some economical traits Kermani sheep. Iranian Journal of Agriculture Science, 33: 395-402 (In Persian).

11. Elfadilli, M., C. Michaux., J. Detilleux and P.L. Leroy. 2000. Genetic parameters for growth traits of the Moroccan Timahdit breed of sheep. Small Ruminant Research, (37): 203-208.

12. Eteghadi, B., N. Ghavi-Hoseinzadeh and A. Shadparvar. 2015. Estimation of genetic parameters of average daily gain and klibre ratio in sheep for Guilan province. Iranian Journal of Animal Science Research, 7: 104-112 (In Persian).

13. Farhadi, G. and H. Roshanfekr. 2016. Estimation of genetic parameters of some of growth traits in Lori-Bakhtiari lambs of Khuzestan province with considering the maternal effects. Journal of Animal Science Research, 26: 151-161 (In Persian).

14. Farhoosh, T. 2004. Investigation of some characteristics of two-veal sheep fleece (ArkhameninusGhezel and Arkhamenius-Moghani). Thesis for Master of Science, Tabriz University. Tabriz. Iran. (In Persian).

15. Gizaw, S., S. Lemma., H. Komen and J.A.M.Van Arendonk. 2007. Estimates of genetic parameters and genetic trends for live weight and fleece traits in Menz sheep. Small Ruminant Research, 70: 145153.

16. Jafaroghli, M., A. Rashidi., M.S. Mokhtari and A.A. Shadparvar. 2010. (Co) Variance companents and genetic parameter estimates for growth traits in Moghani sheep. Small Ruminant Research. 91: 170-177.

17. Jawasreh, K.h., Z. BaniIsmail., F. Iya, V. Castañeda-Bustos and M. Valencia-Posadas. 2018. Genetic parameter estimation for pre-weaning growth traits in Jordan Awassi sheep. Veterinary World, EISSN: 2231-0916.

18. Jiang, D.i., Y. Zhang. K.C. Tian., Lazate., J.F. Liu., X.M. Xu., Y.J. Zhang., T.H. Zhang. 2011. Estimation of (co)variance components and genetic parameters for growth and wool traits of Chinese superfine merino sheep with the use of a multi-trait animal model. Livestock Science, 138: 278-288.

19. Khalili, D., R. VaezTorshizi., A. Shamsi., S. Golkhandan and A. Iranshahi. 2010. Estimation of variance components (covariance) and genetic parameters of production and reproductive traits in Baluchi sheep. Animal Science Journal, 85: 22-30 (In Persian).

20. Khosravi, S., M. Asadi Fozi and A. Esmaili Koshkooyeh. 2018. Evaluation of genetic progress of growth traitsin kermani sheep breeding station. Research on Animal Production, 8(18): 187-193 (In Persian).

21. Latifi, M., S. Alijani., A. Taghizadeh and Gh. Moghaddam. 2014. Estimation of genetic parameters for growth traits in Mehrabani sheep. Journal of Animal Science Research, 24: 1-10 (In Persian).

22. Makarechian, M. 2002. Application of animal genetics in livestock breeding. Shiraz University Press Publishing Center, 580 pp (In Persian).

23. Maria, G.A., K.G. Boldman and L.D. Van Vleck. 1993. Estimates of variances due to direct and maternal effects for growth traits of Romanov sheep. Journal of Animal Science, 71: 845-849.

24. Meyer, K. 2000. DFREML Version 3.1: User notes. 
25. Mohammadabadi, M.R. and R. Sataimokhtari. 2013. Estimation of (co)variance components of ewe productivity traits in Kermani sheep. Slovak Journal of Animal Science, 46: 45-51.

26. Moradi Shahrbabak, H., A.H. Khaltabadi Farahni and H. Mohammadi. 2015. Comparison different models for estimation genetics parameter of growth traits with the likelihood ratio test in Kermani sheep breed. Journal of Animal Science Research, 3: 1-11 (In Persian).

27. Murphy, T.W., Y.M. Berger., P.W. Holman., M. Baldin., R.L. Burgett and D.L. Thomas. 2018. Genetic and non-genetic factors influencing the live weight of artificially-reared lambs. Small Ruminant Research. Accepted Manuscript.

28. Nosrati, M. 1999. Estimation of genetic and phenotypic parameters of body weight at different ages and annual wool production in Moghani sheep. Thesis for Master of Science, Tabriz University. Tabriz. Iran (In Persian).

29. Ozcan, M., B. Ekiz., A. Yilmaz and A. Ceyhan. 2005. Genetic parameter estimates for lamb growth traits and greasy fleece weight at first shearing in Turkish Merino sheep. Small Ruminant Research, 56: $215-222$.

30. Patiabadi, Z., S. Savar Sofla and Sh. Varkoohi. 2017. Estimation of (co) variance components for growth traits of shall sheep using different animal models. Research on Animal Production, 8(17): 149-156 (In Persian).

31. Rahimi, H. 2006. Genetic and phenotypic trends of production and reproductive traits in Lori-Bakhtari sheep. Thesis for Master of Science. Shahrekord University, Shahrekord. Iran, 104 pp (In Persian).

32. Rashedi Dehsahraei, A. 2012. Bayesian Estimation of genetic and phenotypic Parameters of growth traits and wool yield in lori-bakhtiari sheep. Thesis for Master of Science. Agricultural and Natural Resources University of Khuzestan. Ahwaz. Iran. 104 pp (In Persian).

33. Rashedi Dehsahraei, A., J. Fayazi., M. Vatankhah and M.T. Beige nasiri. 2013. Estimation of (Co) variance components and genetic parameters for growth traits in Lori-Bakhtiari lambs using a Bayesian approach via Gibbs sampling. Journal of Ruminant Research, 2: 109- 128 (In Persian).

34. Rashidi, A., M.S. Mokhtari., A. Safi Jahanshahi and M.R. Mohammad Abadi. 2008. Genetic parameter estimates of pre-weaning growth traits in Kermani sheep. Small Ruminant research, 74, pp: 165-171.

35. Robinson, O.W., B.T. Mc Daniel and B.T. Rincon. 1981. Estimation of direct and maternal addition and heterotic effects from crossbreeding of experiment in animal. Journal of Animal Science, 52: 4450 .

36. Rout, P.K., O. Matika., R. Kaushik., M.S. Dige., G. Dass., M.K. Singh and S. Bhusan. 2018. Genetic analysis of growth parameters and survival potential of Jamunapari goats in semiarid tropics. Small Ruminant Research, $165: 124-130$.

37. Saghi, D.A., A.R. Shahdadi., F. Kazemi and K. Mohammadi. 2018. Estimates of covariance functions for growth of Kordi sheep in Iran using random regression models. Small Ruminant Research, 162: 69-76.

38. Savar Sofla, S., Z. Patiabadi., Sh. Varkoohi and M.H. Hadi Tavatori. 2017. Estimation of genetic, phenotypic and environmental trends for growth traits in Shal sheep. Livestock Research (Quarterly), 5: 17-29 (In Persian).

39. Shayesteh, M., M.T. Beige Nasiri and A. Rashedi. 2016. Estimation of (co) variance components and genetic parameters for body weight traits in Zandi sheep using a Bayesian approach. Livestock Research (Quarterly), 5: 13-24 (In Persian).

40. Sheikh Ahmadi, M. 2008. Estimation of Genetic Parameters and Trend Evaluation of Analyzes of a Trait and Multiplicity of Economic Traits in Markhoz Goat. Thesis for Master of Science, sanandaj University. Sanandaj. Iran, 67 pp (In Persian).

41. Talebi, M.A. and M.A. Edriss. 1998. Estimation of genetic and environmental parameters affecting preweaning traits of Lori-Bakhtiari lambs. Iranian Journal of Agriculture Science, 29: 325-333 (In Persian).

42. Tosh, J.J. and R.A. Kemp. 1994. Estimation of Variance components for lamb weights in three sheep population. Journal of Animal Science, 72: 1184-1190.

43. Van Tassell, C.P. and L.D. Van Vleck. 1995. A Manual for Use of MTGSAM. A Set of FORTRAN programs to apply gibbs sampling to animal models for variance component estimation [DRAFT]. U.S. Department of Agriculture, Agricultural Research Service.

44. Van Vleck, L.D. 1993. Selection index and introduction to mixed model methods. C.R.C. Press, Inc. U.S.A.

45. Willham, R.L. 1972. The role of maternal effects in animal breeding. III. Biometrical aspects of maternal effects in animals. Journal of Animal Sciences. 35: 1288-1293. 


\title{
Estimation of Genetic and Phenotypic Variance Components for Body Weight Traits of Moghani Sheep using Gibbs Sampling Approach
}

\begin{tabular}{c} 
Reza Behmaram $^{\mathbf{1}}$ and Azar Rashedi Dehsahraei \\
\hline \hline 1- Assistant professor of Genetics \& Animal Breeding, University of Mohaghegh Ardabili, \\
(Corresponding author: behmaram.reza@ yahoo.ca) \\
2- PhD graduated of Genetics and Animal Breeding, Agriculture \\
and Natural Resources University of Khouzestan \\
Received: April 4, 2019 Accepted: May 5, 2019
\end{tabular}

\begin{abstract}
In this study for prediction of the (Co)-variance components and genetic and phenotypic parameters for growth traits of Moghani sheep, data from birth weight (7368 records), weaning weight (5892 records), 6-month weight (5110 records), 9-month weight (2912 records) and yearling weight (2893 records) were used. Data was collected during 1995-2017 from Moghani sheep breeding station in Jafarabad of Moghan. (Co) variance components and genetic parameters were estimated by Bayesian statistical method based on Gibbs sampling technique, because of high accuracy of bayasian method. Environmental factors such as birth year, birth season, Sex of lamb, birth type and mother's age were significant on all traits $(\mathrm{P}<0.01$ and as fixed effects in the mode. Age of lamb during weaning was used as covariate in the model. Based on lowest AIC for birth weight, weaning weight, 6-month weight, 9-month weight and yearling weight the direct heritability was found to be $0.35 \pm 0.003,0.20 \pm 0.004,0.23 \pm 0.002$, $0.36 \pm 0.004$ and $0.24 \pm 0.003$ for the traits, respectively. Maternal heritability of birth weight and weaning weight were $0.26 \pm 0.002$ and $0.11 \pm 0.002$, respectively. The results revealed by increasing lamb's age and reducing lamb's dependence on mother, the eontribution of maternal effects to direct genetic effects decreases. Therefore, the importance of this effect was reduced by increasing age due to decreased dependenc if lamb to mother. Low to moderate heritability of the traits indicated that selection for these traits will result in a low to moderate genetic improvement.
\end{abstract}

Keywords: Bayesian Method, Growth Traits, Genetic Parameters, Moghani Sheep 